\title{
Impact of the Normal Zone Propagations Velocity of High Temperature Superconducting Coated Conductors on Resistive Fault Current Limiters
}

\author{
D. Colangelo and B. Dutoit
}

\begin{abstract}
The engineering critical current $\left(I_{c}\right)$ of the high temperature superconducting coated conductors (HTS-CCs), today available on the market, is not a uniform parameter and varies significantly along the length of the conductors. Moreover, commercial HTS-CCs have a low normal zone propagation velocity (NZPV). This property, together with the $I_{c}$ inhomogeneity, exposes the HTS-CCs to local thermal instabilities. A crucial challenge for the design of resistive fault current limiters (RFCLs) based on HTS-CCs is to avoid the thermal runaway of the conductors, and in this respect the enhancement of the NZPV is a promising solution. In the recent years, several methods have been proposed and many and various techniques are now available to enhance the NZPV. Whichever will be the best technical solution to improve NZPV of HTS-CCs, our aim is to quantify the impact the enhancement of NZPV will have on the design of RFCLs based on HTS-CCs. For this reason, we used numerical models to analyze the effects of the enhancement of NZPV on the limitation performance of a RFCL integrated in a medium voltage (MV) power grid. In this manuscript, we quantify the benefits the enhancement of the NZPV will have on the next generation of HTS-CC-based RFCLs for MV grids.
\end{abstract}

Index Terms-Power Network Modelling, Fault Current Limiters, High Temperature Superconducting Coated Conductors, Normal Zone Propagation Velocity.

\section{INTRODUCTION}

D UE to the complexity of the manufacturing processes of $R E \mathrm{BaCuO}$-based ( $R E$ stands for different rare-earth elements such as gadolinium, ytterbium, yttrium, etc.) high temperature superconducting coated conductors (HTS-CCs), the critical current $\left(I_{c}\right)$ of commercial HTS-CCs varies significatively around the average critical current $\left(I_{\text {c.av }}\right)$ of the tapes [1]. When commercial HTS-CCs are subjected to an applied over-current waveform close to $I_{c . a v}$, the weak zones of the conductors (characterized by low $I_{c}$ ) might quench to their resistive state. The applied current is locally diverted to the stabilizer layer of the HTS-CCs and sources of heat are formed by Joule heating effect. Commercial HTS-CCs have low thermal diffusivity and their longitudinal normal zone propagation velocity (NZPV) is extremely low (a few tens of $\mathrm{cm} / \mathrm{s}$ ). As a consequence, the heat dissipation remains localized and tapes are exposed to thermal instability.

Manuscript received X Month 2014.

Corresponding author's e-mail: daniele.colangelo@epfl.ch.

D. Colangelo and B. Dutoit are with École polytechnique fédérale de Lausanne, EPFL-SCI-IC-BD , Station 14, 1015 Lausanne, Switzerland.

The research leading to these results has received funding from the European Union Seventh Framework Programme (FP7/2007 - 2013) under grant agreement No. 241285
A common method to reduce the heat generated near the weak zones of the HTS-CCs and to mitigate the local thermal instability, is to increase the thickness of the stabilizer material [2]. The resistance of the stabilizer is lowered, the transport current is rapidly diverted to the stabilizer layer and the heat diffuses faster within the tape. Unfortunately, this solution decreases the series normal state resistance of the HTS-CCs and therefore, when HTS-CCs are used for RFCL purposes, tapes of longer length are required to achieve a given current limitation. A direct consequence is an important increase of the final cost of the device.

An alternative approach to guarantee the local thermal stability is the enhancement of the NZPV of the HTS-CCs. Although the enhancement of the NZPV does not solve the $I_{c}$ inhomogeneity issues, it helps to propagate the normal zones from the sectors where $I_{c}$ is lower to the contiguous zones where $I_{c}$ is higher. Consequently, the power peak densities within the HTS-CCs are sensibly reduced without changing the thickness of the stabilizer layer. In the last years, the enhancement of the NZPV has been extensively studied [3]-[7] and several valid methods have been proposed. Even though, the proposed methods are profoundly different, the common conclusion is that the enhancement of NZPV will represent an important breakthrough for superconducting devices, and in particular for the design of RFCLs.

The discussion on the different techniques to improve NZPV is beyond the scope of our work. The aim of our research is to clarify and to quantify the impact the NZPV enhancement will have on the design of RFCLs. For this reason, we employed our experience in numerical modelling to analyze the impact the increase of the NZPV will have on the design of RFCLs based on HTS-CCs. First, we have modified numerically the NZPV of HTS-CC candidates. All the HTS-CC candidates have the same architecture (similar to the tapes produced by SuperPower inc). Then, we simulated a RFCL designed with HTS-CCs with enhanced NZPV in a busbar coupler configuration. The simulated RFCL has the same specifications of the device developed within the framework of the European project ECCOFLOW [8].

In the current manuscript, we detail the numerical model we used to evaluate the HTS-CC candidates with enhanced NZPV. Afterwards, we report the analysis of the limitation performance of a RFCL for MV power grids designed with HTS-CCs with different NZPVs. We follow with a discussion on the simulation results on the impact the enhancement of 
Electrical Part
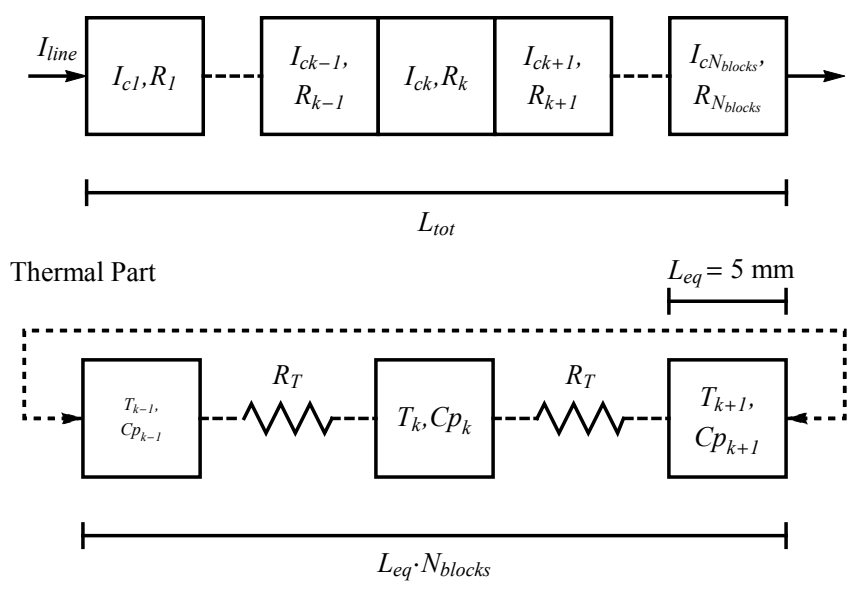

Fig. 1. A schematic overview of the electrical and the thermal part of the numerical model. In the electrical part, the longitudinal length of each tape ( $L_{t o t}$ ) is divided in $k$ blocks of length $L_{k}$. The thermal part of the model is solved considering a limited portion of tapes equal to $L_{e q} \cdot N_{\text {blocks }}$ and the solution is repeated along the whole length $L_{t o t}$. The thermal conduction between the different part of the tape is implemented through the thermal resistance $R_{T}$. Each block implements the same cross-section architecture.

NZPV will have on the design of HTS-CC-based RFCLs.

\section{OVERVIEW OF THE NUMERICAL MODEL}

Each phase of a RFCL device is composed by as many parallel HTS-CCs as needed to obtain the required total critical current $\left(I_{c . t o t}\right)$. Nevertheless, for simplicity, our model does not consider differences between the paralleled HTS-CCs and, therefore, a single HTS-CC is simulated. As shown in Fig. 1, for the electrical part of the model, the longitudinal length of each paralleled tape $\left(L_{t o t}\right)$ is divided in $N_{\text {blocks }}$ isothermal series blocks.

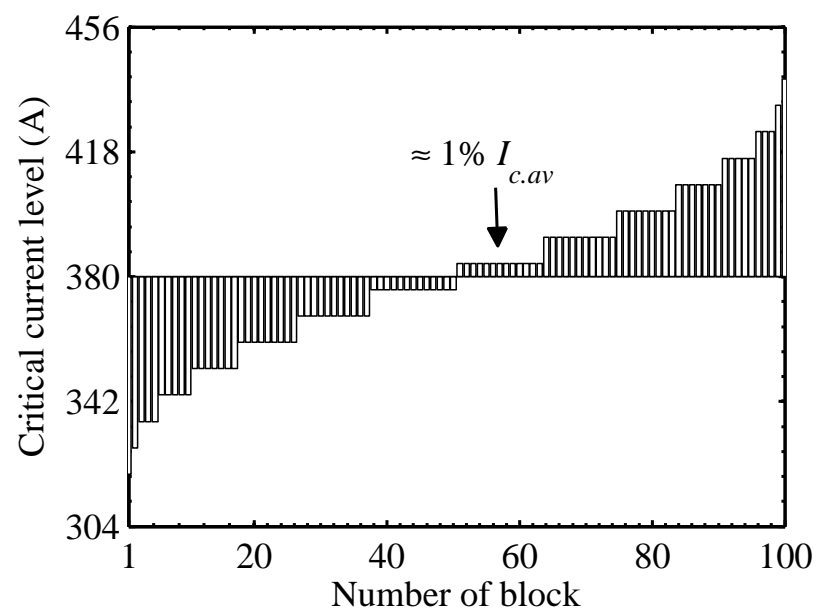

Fig. 2. Critical current levels implemented in the simulated HTS-CCs. The discrete array of $I_{c}$ levels derives from a Gaussian distribution characterized by an average critical current of circa $380 \mathrm{~A}$ and a standard deviation over length of $6.59 \%$.
The model enables us to consider Gaussian distributions of $I_{c}$ derived from experimental measurements. The Gaussian distribution we considered in our example is characterized by an average value of $380 \mathrm{~A}$ and a standard deviation over length $(\sigma)$ equal to $6.59 \%$. As explained in details in our previous work [9], the array of critical current levels (G1) that represents the Gaussian distributions of $I_{c}$ takes into account the critical current levels and their corresponding probability. In our example, the Gaussian distribution is discretized in 16 levels of $I_{c}$, and the probability assigned to each $I_{c}$ level is given by the number of blocks with the same level of $I_{c}$ (Fig. 2)

Even if in principle, it is possible to implements any number of blocks we limited $N_{\text {blocks }}$ to 100 . With higher $N_{\text {blocks }}$ the computation time becomes extremely important and therefore the value we chose is a compromise between the accuracy (on the discretization of the Gaussian distributions of $I_{c}$ ) and an acceptable computation time. Due to the thermal calibration of the model, for the thermal part, the length of each block is fixed to $L_{e q}=5 \mathrm{~mm}$. As in real applications a single HTSCC is between 100 and $300 \mathrm{~m}$, this would imply a number of blocks larger than 20000 . In order to limit $N_{\text {blocks }}$ to 100 , we calculate the thermal conduction dynamics in a reduced part of the tape and we assume that this part is periodically repeated along the whole tape length $L_{t o t}$. As the number of blocks is fixed to 100 , whichever is $L_{t o t}$, the thermal part of the model is solved on $50 \mathrm{~mm}$ and repeated for the whole $L_{t o t}$.

The heat exchanged by two adjacent portions of the simulated tape (blocks) is taken into account through the thermal resistance $R_{T}$ (Fig. 1). The model does not consider the heat exchange between the HTS-CC and the liquid nitrogen bath (adiabatic assumption) where the tape is immersed. Furthermore, the layers of the HTS-CC simulated by the model are: the substrate (hastelloy C-276), the superconducting material and the stabilizer (silver layer) [10]. The buffer layer is then neglected.

The model is developed in Simulink ${ }^{\circledR}$ environment and can be interfaced with common circuit elements. It can be customized for any type of HTS-CC but the calculations presented in the current manuscript are performed using specific characteristics of $12 \mathrm{~mm}$ wide HTS-CCs manufactured by SuperPower Inc. More details about the numerical model are reported here [11].

\section{A. A formulation of the power index temperature dependence}

The onset of the transition to the normal state of the superconducting material, can be modeled using a phenomenological power law which derives from current-voltage measurements [12]. The power law describes the macroscopic effects of a superconducting material far below $I_{c}$ and, in principle, it loses validity when the applied current exceeds the critical current of the tape [13]. However, implementing a variable power index ( $n$-value) makes possible to use it to describe the superconducting transition in over-current regimes and, eventually, to the normal state regime [14].

In this respect, we used a particular form of the power law where we defined a variable $n$-value to model the resistivity 
transition of the superconducting material. We model the normal state resistivity $\left(\rho_{\text {norm }}\right)$ of the superconducting material using data taken from the literature [15]. The whole superconducting to normal state resistivity of the superconducting material is given by

$\rho_{s c}= \begin{cases}\frac{E_{c}}{J_{c}(T)}\left(\frac{\left|J_{s c}\right|}{J_{c}(T)}\right)^{(n(T)-1)}, & T<T_{c} \\ \rho_{\text {norm }}(T), & T \geq T_{c} \text { or } \rho_{p l} \geq \rho_{\text {norm }}\end{cases}$

where $E_{c}$ is an electrical field criterion equal to $1 \mu \mathrm{V} / \mathrm{cm}$, $J_{s c}$ is the current density through the superconducting material, $J_{c}$ and $n$ are, respectively, the critical current density and the power index of the modelled HTS-CC. The critical current density $J_{c}$ decreases linearly with the temperature increase and it corresponds to the transport current $\left(I_{s c}\right)$ divided by the superconductor cross-section.

$$
J_{c}(T)=J_{c}\left(T_{0}\right)\left(\frac{T_{c}-T}{T_{c}-T_{0}}\right), \quad T<T_{c}
$$

where $T_{0}$ is the temperature of the cooling bath far from the sample $(77 \mathrm{~K}$ at $1 \mathrm{~atm})$ and $T_{c}$ is the critical temperature of the tape $(\approx 92 \mathrm{~K})$. The empirical temperature dependence of the transition index we implement in our numerical model is the following one

$$
n= \begin{cases}k 1\left[\frac{\chi T_{0}-T}{\chi T_{0}-T_{0}}+1\right]+1, & T \leq \chi T_{0} \\ k 1\left(\frac{T_{c}-T}{T_{c}-\chi T_{0}}\right)^{\beta}+1, & \chi T_{0}<T \leq T_{c}\end{cases}
$$

where the value $\beta$ determines the shape of the temperature dependence of the $n$-value. The parameters $k 1$ is equal to $\left(n_{0}-1\right) / 2$ where $n_{0}$ is the average transition index obtained from the producer datasheet.

As shown in Fig. 3, the temperature dependence is divided in three regimes. In the first regime, the dissipation of power is extremely low and the $n$-value varies linearly with the temperature [16]. The parameter $\chi$ defines the transition between the first and the second regime of the characteristic. As soon as the losses become important $(T>\chi T)$, the function switches to the second regime and the transition index falls down from circa half of its initial value to 1 [17]. In our case, the parameter $\beta$ is equal to 0.25 that is a common value used to model the over-current resistivity transition to normal state [18], [19].

We faced our model against four points over-current measurements. A sinusoidal voltage drop of $600 \mathrm{~V}$ (peak) was applied to a HTS-CC of $8 \mathrm{~m}$ of length and the transport current was set through a variable resistance in series to the tape. The measures were performed at Karlsruhe Institute of Technology (KIT) and shared in the framework of the ECCOFLOW project. Two examples of comparison between the model and experimental measurements are shown in Fig. 4a-b. The limited current (blue solid line in Fig. 4a-b) measured during the experiences, is used as input in the numerical model. In the two examples (where the limited current is different), with our

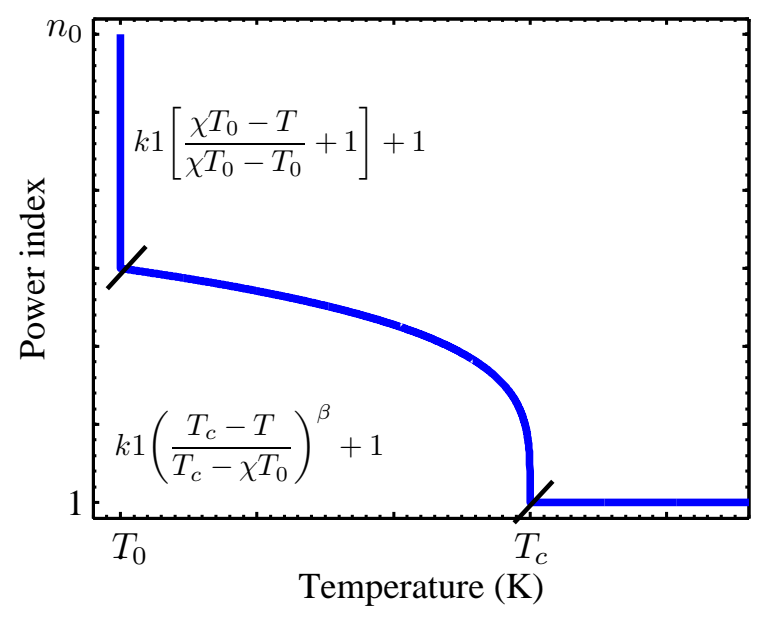

Fig. 3. The temperature dependence of the transition index implemented in the numerical model. The power index decreases with the temperature and it tends to 1 approaching $T_{c}$. The initial value of the power index is equal to 31 and $\beta$ is equal to 0.25 .

implementation of the temperature dependence of the power index, the simulated voltage drop across the total length of the HTS-CC (black dashed line in Fig. 4a-b) reproduces with good agreement the voltage drop measured in the experiments (red dotted line in Fig. 4a-b). The thickness of the composite materials of the simulated HTS-CC are respectively, 104.5 $\mu \mathrm{m}, 1 \mu \mathrm{m}$ and $4.8 \mu \mathrm{m}$ for the substrate, the superconducting material and the stabilizer layer.

\section{B. Thermal conduction and estimation of the NZPV}

The heat shared between the different blocks of the simulated HTS-CC is modeled through a thermal resistance $\left(R_{T}\right)$ proportional to the thermal conductivity of the tape. The equivalent resistance $R_{T}$ comes from the paralleled thermal resistances of the different layers that compose the HTS-CC. The $R_{T}$ is modelled as follows

$$
R_{T}=\frac{1}{\alpha_{N Z P V}} \cdot \sum_{j=1} \frac{L_{t h}}{k_{j}(T) \cdot A_{j}}
$$

where $k_{j}$ and $A_{j}$ are, respectively, the thermal conductivity and the cross section of the $j_{t h}$ layer of the block $k$ [20], [21].

The parameter $L_{t h}$ is needed in order to have a consistent (in term of unit) $R_{T}$ inversely proportional to both the thermal conductivity and the cross-section of the tape.

The value of $R_{T}\left(L_{t h}\right)$ is determined facing the model we developed in Simulink ${ }^{\circledR}$ (lumped element model) against an equivalent model based on finite element method (FEM) techniques. If we consider the superconducting layer as a homogenous material and we consider only the longitudinal gradient of temperature, we can calculate the rate of heat conduction between two generic point solving the Fourier's law in its one-dimensional form.

This easy concept can be scaled to our lumped element model. In the FEM model, the temperature in center of the superconducting layer is recorded with virtual probes each 

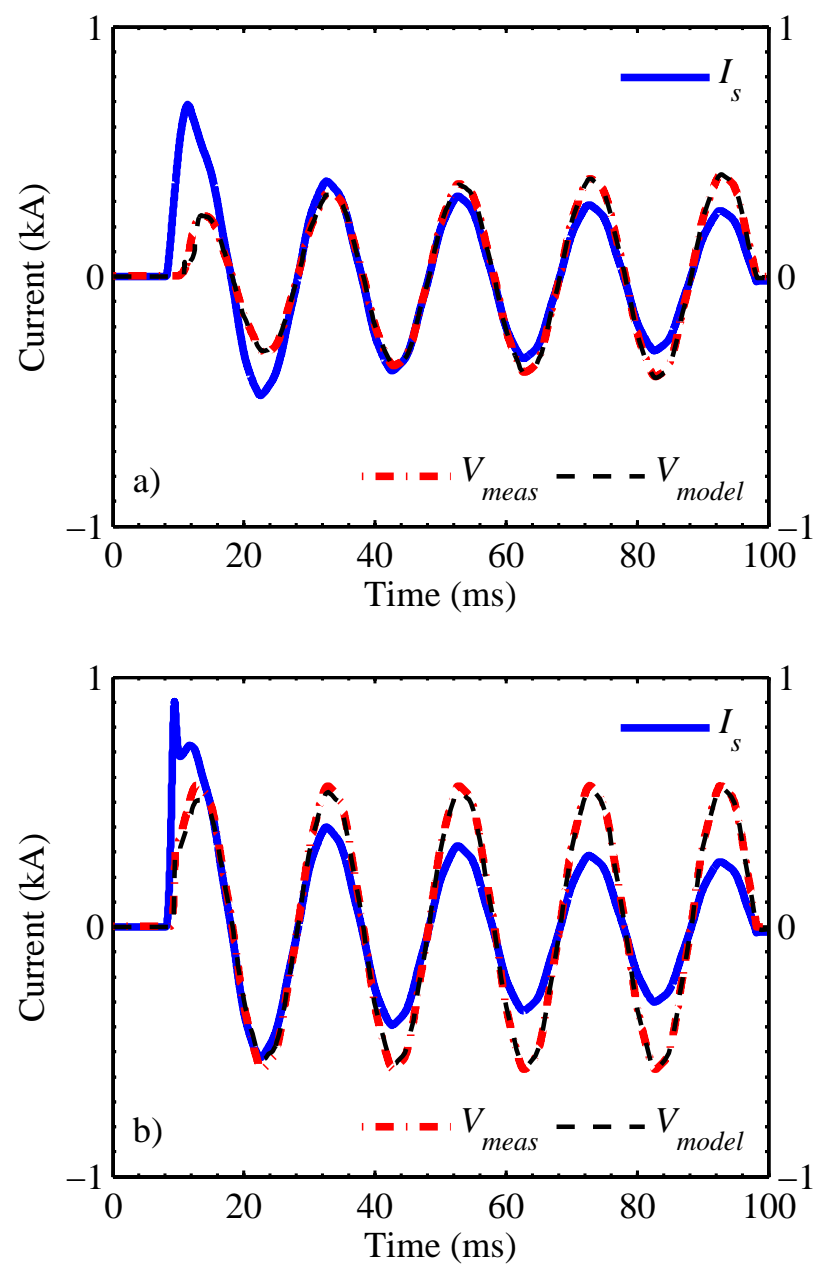

Fig. 4. Comparison between the numerical model and experimental measurements. The measured transport current $\left(I_{s}\right)$ is applied as input to the model. In both cases, where the applied current is different, with our formulation of the temperature dependence of the power index, the model reproduces ( $V_{\text {model }}$ ) the experimental voltage drop $\left(V_{\text {meas }}\right)$ with good approximation.

$5 \mathrm{~mm}$. In our lumped element model, we assume that the temperature of each block is an average temperature equal to the local temperature tracked by a corresponding virtual probe in the FEM model. The value of $L_{t h}$ is the value that minimize the error between the FEM model and the lumped element model. The details of the calibration of the thermal part of the model are reported here [11].

The thermal resistance $R_{T}$ can be varied through the parameter $\alpha_{N Z P V}$. In our model, the variation of $\alpha_{N Z P V}$ corresponds to a modification of the heat exchange between the blocks. For different values of $\alpha_{N Z P V}$, the NZPV is calculated simulating an over-current pulse as input of the model. The simulated HTS-CC is divided in 100 blocks and, as shown in Fig. 5, the quench is initiated assigning a lower value of $I_{c}$ to the middle blocks.

The voltage drops across the different blocks can be easily recorded and the NZPV immediately calculated. The example of Fig. 6 illustrates the time evolution of the voltage drops of block 67, block 68 and block 69 (B67, B68 and B69

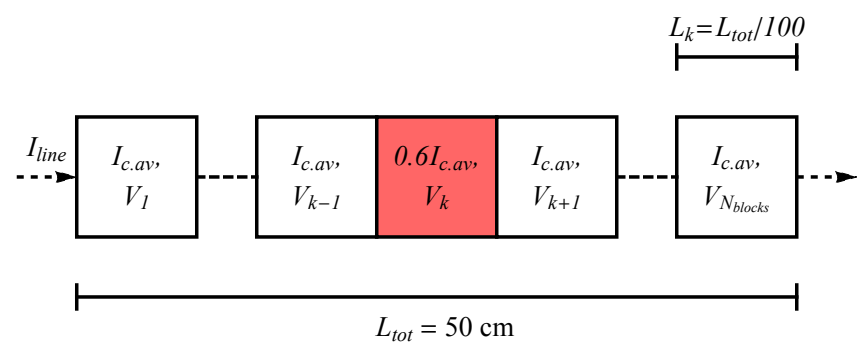

Fig. 5. Sketch of the $I_{C}$ inhomogeneity distribution used to calculate the NZPV as a function of the parameter $\alpha_{N Z P V}$. The simulated HTS-CC is divided in 100 blocks and the total length of the simulated HTS-CC $\left(L_{t o t}\right)$ is $50 \mathrm{~cm}$. The length of a single length $\left(L_{k}\right)$ is $5 \mathrm{~mm}$.

in Fig. 6) obtained with a current pulse of $1.2 I_{\text {c.av }}$. The thickness of the composite materials of the simulated HTS$\mathrm{CC}$ are respectively, $104.5 \mu \mathrm{m}, 1 \mu \mathrm{m}$ and $4.8 \mu \mathrm{m}$ for the substrate, the superconducting material and the stabilizer layer. The NZPV of each HTS-CC candidates can be calculated using the following expression

$$
N Z P V=\frac{L_{k}}{t_{2}-t_{1}}=\frac{0.5}{t_{2}-t_{1}}
$$

where $L_{k}$ is the length of block $k$ equal, in this particular case, to $5 \mathrm{~mm}$ for each block. The relevant times $t_{1}$ and $t_{2}$ are defined using an arbitrary quench criterion (Fig. 6).

Figure 7 shows the relation between the parameter $\alpha_{N Z P V}$ and the resulting NZPV. The NZPV varies sensibly with the thickness of the stabilizer layer $\left(t h_{A g}\right)$ because with thinner $t h_{A g}$ the tape is more resistive (in normal state) and, with a fixed current source, higher is the power dissipated within the tape. With $\alpha_{N Z P V}=1$ and a current pulse of $1.2 I_{\text {c.av }}$, the NZPV is circa $20 \mathrm{~cm} / \mathrm{s}$ for $t h_{A q}$ equal to $1.5 \mu \mathrm{m}$. Varying the thermal resistance $R_{T}$, through the parameter $\alpha_{N Z P V}$, the NZPV is numerically modified starting from actual NZPV of the commercial HTS-CCs (tens of $\mathrm{cm} / \mathrm{s}$ ).

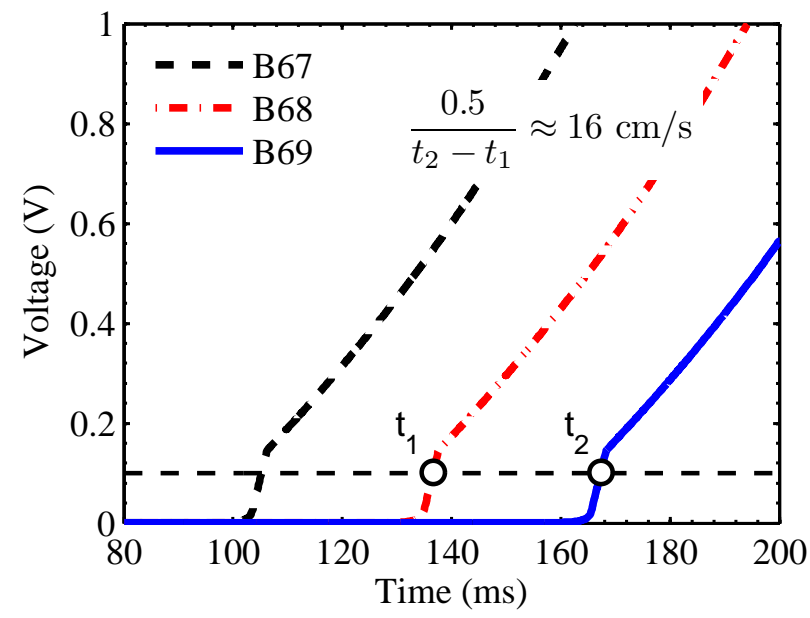

Fig. 6. Evolution of the simulated voltage tracks with a stabilizer layer of $4.8 \mu \mathrm{m}$ thick, $\alpha_{N Z P V}=1$ and using as input a current pulse of $1.2 I_{\text {c.av }}$. The estimated NZPV of the simulated HTS-CC is $\approx 16 \mathrm{~cm} / \mathrm{s}$. 


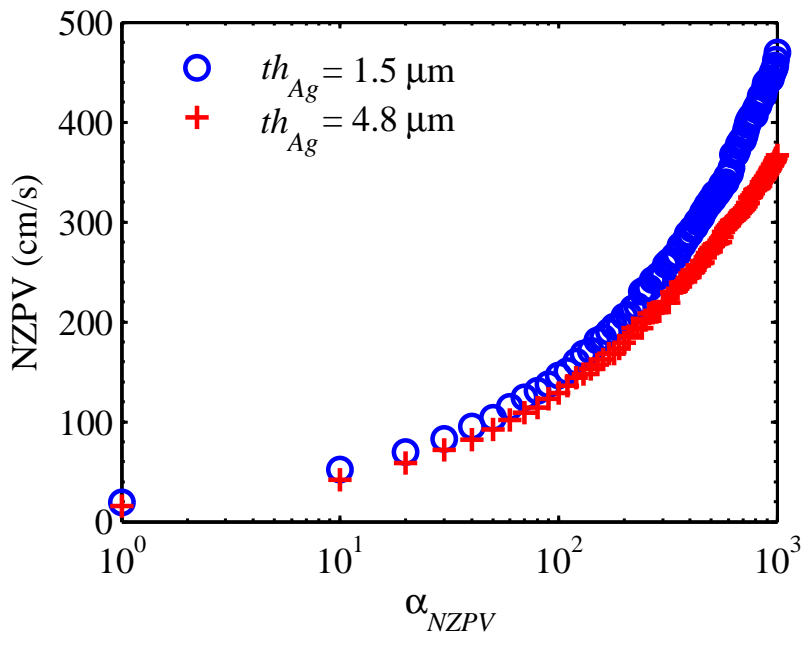

Fig. 7. Relation between the parameter $\alpha_{N Z P V}$ and the simulated normal zone propagation velocity for given thickness of stabilizer layer and an applied current pulse of $1.2 I_{\text {c.av }}$. With $t h_{A g}=1.5 \mu \mathrm{m}$ the initial NZPV is $\approx 20 \mathrm{~cm} / \mathrm{s}$

\section{ANALYSIS OF THE NZPV ENHANCEMENT}

We have simulated a three phases RFCL module with 5 parallel HTS-CCs per phase. The simulated RFCL has the same specifications as the limiter developed within the European project ECCOFLOW. Similarly to our previous work [22], the RFCL is integrated into a MV grid where it operates in busbar coupling configuration. The MV grid is characterized by the real parameters of a HV/MV substation operated by the Spanish utility company Endesa [23] on the Balearic island of Mallorca. Our simulation reproduces not only the ECCOFLOW device but also the grid conditions where the device will be tested.

The single line diagram of the RFCL device integrated in the Endesa substation is shown in Fig. 8. The prospective shortcircuit current $\left(I_{p s s c}\right)$ can be set through a fault resistance

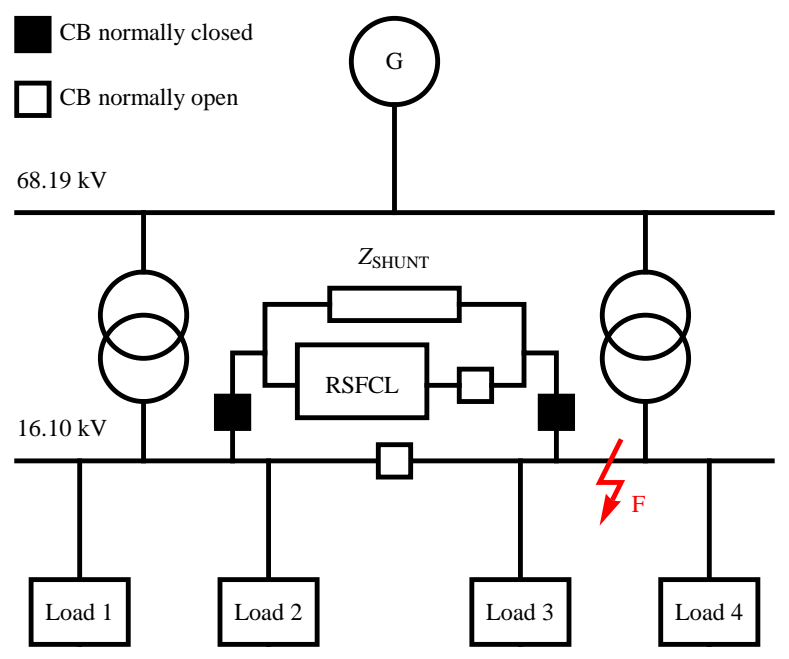

Fig. 8. Single line diagram of the busbar coupling application of the modelled RFCL device. The diagram shows the short-circuit location (F). The modelled RFCL device has the same specifications and the same grid conditions of the ECCOFLOW prototype.
$\left(R_{F}\right)$ inserted in the fault location (point $\mathrm{F}$ in Fig. 8). The specifications of the limiter are summarized in Table I.

TABLE I

FAULT CURRENT LIMITER PARAMETERS

\begin{tabular}{l|c|c}
\hline Parameter & Expression & Description \\
\hline \hline$U_{0}$ & $24 \mathrm{kV}$ & Nominal voltage \\
$I_{n, R M S}$ & $1 \mathrm{kA}$ & Nominal current \\
$I_{\text {C.tot }}$ & $1.9 \pm 0.2 \mathrm{kA}$ & Total critical current \\
$Z_{\text {shunt }}$ & $1.4 \Omega$ & External shunt impedance \\
$t_{C B_{H T S}}$ & $80 \mathrm{~ms}$ & $C B_{H T S}$ tripping time \\
$t_{\text {fault }}$ & $1 \mathrm{~s}$ & Maximum fault time \\
$T_{\text {max }}$ & $360 \mathrm{~K}$ & Max temperature allowed \\
\hline
\end{tabular}

In order to avoid the local thermal instability of the HTS$\mathrm{CCs}$, the ECCOFLOW prototype was manufactured using tapes $180 \mathrm{~m}$ long (length of a single tape per phase, $L_{t o t}$ ) and stabilized with an equivalent silver layer thickness $\left(t h_{A g}\right)$ of $3.5 \mu \mathrm{m}$.

In the simulations reported in this manuscript, the thickness of the silver stabilizer is fixed to $1.5 \mu \mathrm{m}$ and the length of the paralleled HTS-CCs (length of a single tape per phase) is chosen in order to have the same resistance offered by a tape with $L_{t o t}=180 \mathrm{~m}$ and $t h_{A g}=3.5 \mu \mathrm{m}$.

For any thickness of stabilizer, it is easy to obtain the resistance per unit of length $\left(R_{p u l}\right)$. Then, it is possible to estimate the equivalent resistance, offered by a HTS-CC during the limitation of a short-circuit $\left(R_{i n t}\right)$, integrating $R_{\text {pul }}$ (Fig. 9) over the interval of temperature as follows

$$
R_{\text {int }}=\frac{1}{T_{\max }-T_{c}} \int_{T_{c}}^{T_{\max }} R_{p u l}(T) d T
$$

where $T_{\max }$ is the maximum admissible temperature (360 $\mathrm{K})$. Afterwards, it is extremely simple to calculate which $L_{t o t}$ gives the same resistance given by $L_{t o t}=180 \mathrm{~m}$ and $t h_{A g}$ $=3.5 \mu \mathrm{m}$. In the case of $t h_{A g}=1.5 \mu \mathrm{m}$, we obtain a single tape per phase equal to $100 \mathrm{~m}$. Keeping fixed $t h_{A g}=1.5 \mu \mathrm{m}$ and $L_{t o t}=100 \mathrm{~m}$, we varied numerically the NZPV of HTSCCs from $\approx 20 \mathrm{~cm} / \mathrm{s}$ up to $\approx 500 \mathrm{~cm} / \mathrm{s}$. The summary of the performed simulations is reported in Table II.

TABLE II

SUMMARY OF THE SIMULATION PARAMETERS

\begin{tabular}{l|c|c}
\hline Parameter & Value & Description \\
\hline \hline$t h_{A g}$ & $1.5 \mu \mathrm{m}$ & Thickness silver stabilizer \\
$L_{t o t}$ & $100 \mathrm{~m}$ & Length of a single HTS-CC \\
$N Z P V$ & from $\approx 20 \mathrm{up} \mathrm{to} \approx 500 \mathrm{~cm} / \mathrm{s}$ & Normal zone prop. velocity \\
\hline
\end{tabular}

\section{A. Tape with low NZPV}

The $I_{p s s c}$ on the busbar line is set varying the fault resistance $R_{F}$ at the short-circuit location $\mathrm{F}$ (Fig. 8). When the fault is simulated with relatively high value of $R_{F}$ (low values of $I_{p s s c}$ ), the zones/blocks of the simulated HTS-CC that are characterized by low $I_{c}$ quench and limit the shortcircuit current through the device.

In case of low silver stabilization (e.g. $t h_{A g}=1.5 \mu \mathrm{m}$ ), the few quenched zones will introduce in the grid their resistance 


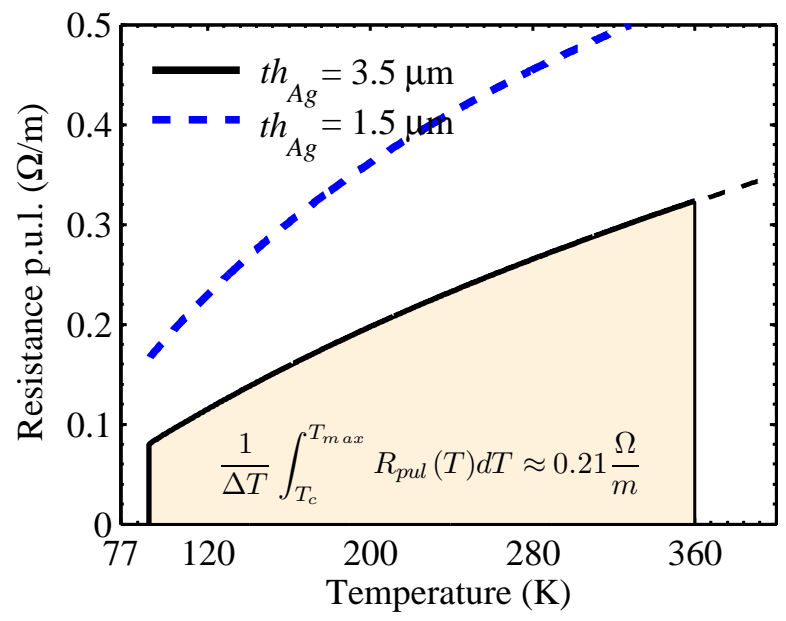

Fig. 9. Normal state resistance per unit length of a HTS-CC in case of a stabilizer thickness of $3.5 \mu \mathrm{m}$ (solid black line) and a stabilizer thickness of $1.5 \mu \mathrm{m}$ (dashed blue line). In the range between $T_{c}$ and $T_{\max }$, the $R_{p u l}$ is used to calculate the $L_{t o t}$ of the simulated HTS-CCs.

(black dashed line of Fig. 10a) and the limited current through a single parallel HTS-CC might fall below $I_{c . a v}$.

As in this case the HTS-CCs are characterized by low $\mathrm{NZPV}$, the heat developed in the quenched parts of the tapes by Joule heating effect does not evacuate and the zones with higher $I_{c}$ do not contribute to the limitation of the fault.

As a consequence, the short-circuit current is limited by a small portion of the paralleled HTS-CCs (black dashed curve of Fig. 10b) and the temperature increase is mainly hindered by the heat capacity of the quenched portions of the HTSCCs (limiting length, $L_{l i m}$ ). If the amount of the HTS-CCs involved in the limitation of the fault is too small, in some points of $L_{\text {lim }}$ the temperature could exceed $360 \mathrm{~K}$ before the intervention of protection circuit breaker $(80 \mathrm{~ms}$ in our simulations). The evident consequence is the local thermal instability of the parallel HTS-CCs of the RFCL module.

\section{B. Tape with enhanced NZPV}

The NZPV has no influence on the $I_{c}$ inhomogeneity. Therefore, under low value of $I_{p s s c}$, even with higher NZPV, the zones of the HTS-CCs characterized by low critical current $\left(I_{c}<0.9 I_{\text {c.av }}\right)$ will quench limiting the fault current through the paralleled tapes. Unlike the previous case (low NZPV), the heat developed in the sectors quenched to the normal state propagates to the contiguous zones with higher $I_{c}$. The total resistance of the RFCL is higher (red dashed line and blue solid line of Fig. 10a) but the portion of tape which is involved in the limitation of the fault current is now much longer (red dashed line and blue solid line of Fig. 10b). A higher heat capacity contributes to the limitation of the fault current. The amount of energy released locally during the fault is lower than the maximum energy the tape can withstand.

As shown in Fig. 11, with $R_{F}=0$, the maximum admissible temperature criterion is respected independently by the NZPV of the HTS-CC candidates. Extending the analysis to $R_{F}>$ 0 and keeping the parameter $\alpha_{N Z P V}=1$ (corresponding to a
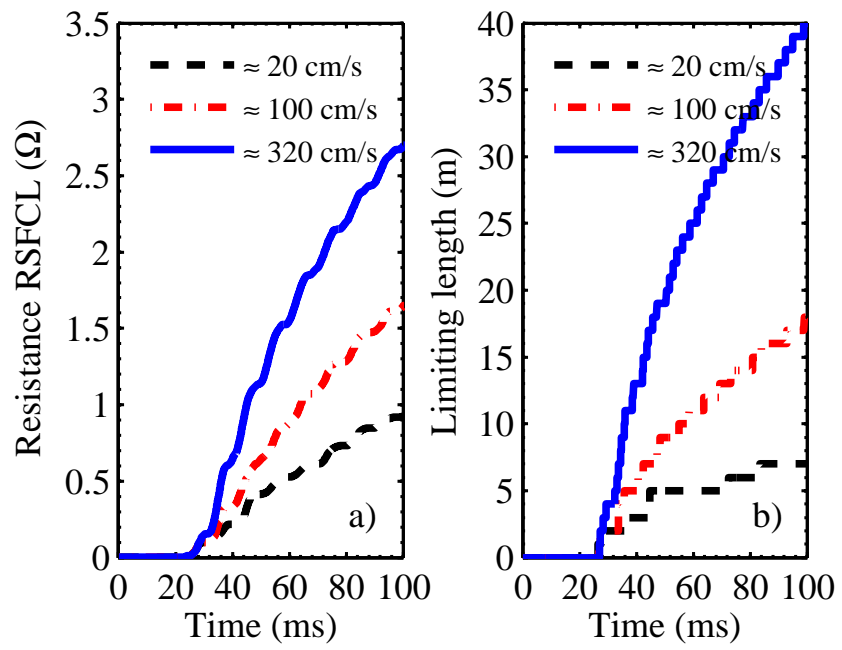

Fig. 10. Resistance profile of the device (a) and limiting length of a single HTS-CC (b) with $R_{F}=2 \Omega$ and a stabilizer thickness of $1.5 \mu \mathrm{m}$. The portion of tape involved in the limitation of the fault current $L_{l i m}$ is proportional to the NZPV of the tape.

$\mathrm{NZPV} \approx 20 \mathrm{~cm} / \mathrm{s}$ ), it is evident that HTS-CCs, $100 \mathrm{~m}$ long with $t h_{A g}=1.5 \mu \mathrm{m}$, are subjected to local thermal instability and a dangerous range of possible $I_{p s s c}$ is left (black dashed line of Fig. 11). Instead, the local stability of such HTS-CCs can be achieved improving the NZPV. With a NZPV grater than $320 \mathrm{~cm} / \mathrm{s}$, the final temperature of the tapes is below 360 $\mathrm{K}$ and the local thermal stability is extended to low values of $I_{p s s c}$ (blue solid line of Fig. 11).

\section{CONCLUSION}

The aim of this research is to investigate what will be the impact of the enhancement of the NZPV of HTS-CCs on the design of RFCLs for MV power grids. We have modified numerically the NZPV of potential HTS-CC candidates for

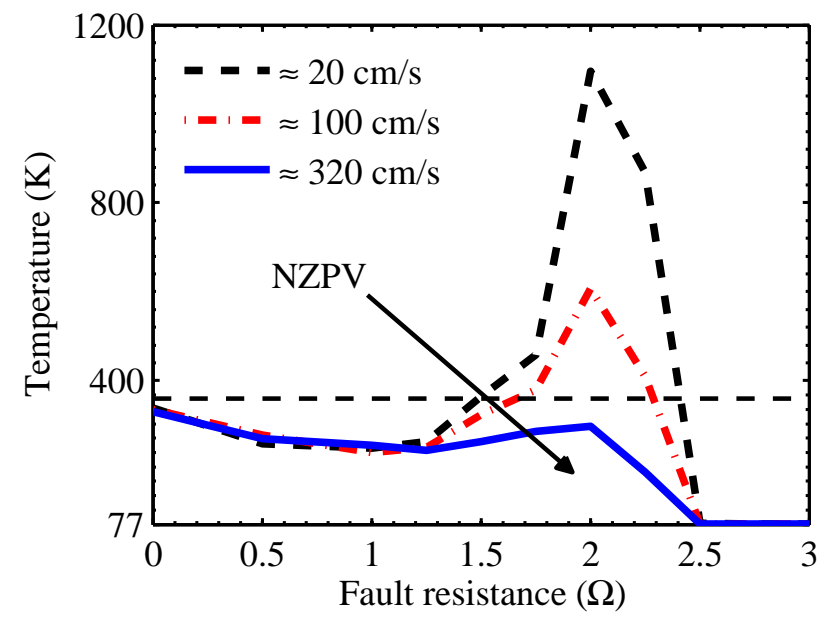

Fig. 11. Final temperature of the weakest zone of the tape function of the fault resistance $R_{F}$. With a current inhomogeneity distribution G1 and $t h_{A g}$ $=1.5 \mu \mathrm{m}$, a NZPV of $320 \mathrm{~cm} / \mathrm{s}$ can guarantee the thermal stability for the whole range of short-circuit currents. 
RFCL applications keeping fixed the thickness of the stabilizer and the length of the tape. Then, we have used our experience in numerical modelling to analyzed the performance of a RFCL with the same specifications of the device developed in the framework of the ECCOFLOW project. The local thermal stability of the HTS-CCs of the ECCOFLOW prototype was achieved designing the limiter with tapes $180 \mathrm{~m}$ long, stabilized with a thick stabilizer layer of $3.5 \mu \mathrm{m}$ and a NZPV in the order of few tens of $\mathrm{cm} / \mathrm{s}$ (value of the actual commercial HTSCCs). The choice of a thick stabilizer is extremely conservative and provides the needed thermal stability but, at the same time, tapes of long length are required to achieve a given current limitation. A higher NZPV will not solve the $I_{c}$ inhomogeneity of the HTS-CCs but it will enable us to design the next generation of RFCLs with less stabilized HTS-CCs, and consequently shorter tapes. With our numerical model, we can quantify the real impact of NZPV on the design of RFCLs. For instance, with a NZPV greater than $320 \mathrm{~cm} / \mathrm{s}$, the amount of HTS-CCs needed for the safe design of RFCLs, with the characteristics of the ECCOFLOW prototype, could be reduced of the $40 \%$. We believe the confirmation of such results and the implementation of techniques able to enhance the NZPV on the manufacturing processes of $12 \mathrm{~mm}$ wide HTS-CCs, will represent an important breakthrough in the design of RFCLs for MV applications.

\section{REFERENCES}

[1] SuperPower.inc FCL Application SuperPower 2G HTS Wire Spec Sheet [Online]. http: \\www.superpower-inc.com.

[2] Fu Y., Tsukamoto O. and Furuse M., "Copper stabilization of YBCO coated conductor for quench protection", IEEE Trans. Appl. Supercond., vol. 13, no. 2, pp. 1780-1783, Jun. 2003.

[3] Badel A. et al., "Hybrid model of quench propagation in coated conductors for fault current limiters", Supercond. Sci. Technol. vol. 25 art. no. 095015, 2012.

[4] Antognazza L. et al., "Comparison between the behavior of HTS thin film grown on sapphire and coated conductors for fault current limiter applications", IEEE Trans. Appl. Supercond., vol. 19, no. 3, pp. 1960-1963, Jun. 2009.

[5] Roy F. et al., "Numerical studies of the quench propagation in coated conductors for fault current limiters," IEEE Trans. Appl. Supercond., vol. 19, no. 3, pp. 2496-2499, Jun. 2009.

[6] Lacroix C. et al., "Normal zone propagation velocity in 2G HTS coated conductor with high interfacial resistance", IEEE Trans. Appl. Supercond., vol. 23, no. 3, 2013.

[7] Levin G. A., Novak K. A., and Barnes P. N., "The effects of superconductor-stabilizer interfacial resistance on the quench of a current carrying coated conductor", Supercond. Sci. Technol., vol. 23, no. 1, p. 014021, Jan. 2010.

[8] ECCOFLOW website [Online]. http://www.eccoflow.org.

[9] Colangelo D. and Dutoit B., "Inhomogeneity Effects in HTS Coated Conductors Used as Resistive FCLs in Medium Voltage Grids", Supercond. Sci. Tech., vol. 25, 095005, 2012.

[10] Park D.K. et al.,'Experimental and numerical analysis of high resistive coated conductor for conceptual design of fault current limiter", Cryogenics, vol. 49, no. 6, pp. 249-253, 2009.

[11] Colangelo D. , "Modelling of 2G HTS coated conductors for fault current limiter applications", pp. 28-31, Ph.D. Thesis EPFL 5916, Lausanne, 2013.

[12] Zeldov E. Amer N.M. Koren G. Gupta A. and McElfresh M.W. and Gambino, "Flux creep characteristics in high-temperature superconductors" Appl. Phys. Lett., vol. 56 no. 7, pp. 680-682, 1990.

[13] Brandt H. E., "Superconductor disks and cylinders in an axial magnetic field. I.Flux penetration and magnetization curves", Phys. Rev. B vol. 58 no. 10 , pp. 6506-6522. 1998.

[14] Paul W. et al., "Fault current limiter based on high temperature superconductors - Different concepts, test results, simulations, applications", Physica C, vol. 354, no. 1-4, pp. 27-33, 2001.
[15] Lortz R. et al., "On the origin of the double superconducting transition in overdoped YBa2Cu3Ox", Physica C, vol. 434, pp. 194-198, 2005.

[16] Berger K. et al., "Influence of temperature and/or field dependences of the E-J power law on trapped magnetic field in bulk YBaCuO", IEEE Trans. Appl. Supercond., vol. 17, no. 2, June 2013.

[17] Angeli G et al.Electrical and Thermal Characterization of Commercial Superconducting YBCO Coated Conductors IEEE Trans. Appl. vol. 23 no. $6602304,2013$.

[18] Lacroix C. and Sirois F., "Concept of a current flow diverter for accelerating the normal zone propagation velocity in 2G HTS coated conductors", Supercond. Sci. Technol., vol. 27, no. 3, 2014.

[19] Roy F., "Modelling and characterization of coated conductors applied to the design of superconducting fault current limiters", pp. 42-43, Ph.D. Thesis EPFL 4721, Lausanne, 2010.

[20] Handbook of Chemistry and Physics, 12-42, [Online]. http://www.hbcpnetbase.com

[21] Lu J, Choi E S and Zhou H D 2008 Physical properties of Hastelloy ${ }^{\circledR}$ C-276 ${ }^{T M}$ at cryogenic temperatures J. Appl. Phys. 103064908

[22] Colangelo D. and Dutoit B., "MV power grids integration of resistive fault current limiter based on HTS-CCs", IEEE Trans. Appl. Supercond., vol. 23 no. 3 art. no. 6378404, 2013.

[23] Endesa website [Online] Available: http://www.endesa.com. 